\title{
Properties of an ultradian biological clock regulating sensory perception in man: A longitudinal study
}

\author{
JOHN W. LOVETT DOUST and INTS PODNIEKS \\ Research Section of Psychophysiology, Clarke Institute of Psychiatry \\ University of Toronto, 250 College Street, Toronto, Canada
}

\begin{abstract}
A neural clock regulating sensory perception is postulated to account for the spontaneous sinewave cycles describing the time series of repetitive critical flicker fusion thresholds monitored at 60-sec intervals. Repeating these tests longitudinally in healthy subjects and in psychiatric patients for a total of 452 observations, 306 spontaneous oscillations emerged. This enabled a series of possibly interfering variables to be evaluated. The perceptual clock is endogenous, innate, selfsustaining, and dependent upon neural integrity. It has a small variance and is independent of body temperature. It is independent of feelings, emotions, and most drugs. The phase of the clock is capable of being reset by challenging attention.
\end{abstract}

A clock is an instrument measuring the passage of time. Timing devices have been described in plants, animals, and man which essentially provide similar information and hence may be called biological clocks. Light is perhaps the most important Zeitgeber or synchronizer of the biological rhythms in plants and animals; in man, however, his social contexts would appear to be just as important (Aschoff, 1967; Luce, Note 1).

We have recently described a biological clock regulating sensory perception which depends for its timing on the integrity of the nervous system (Podnieks \& Lovett Doust, 1975; Lovett Doust \& Podnieks, 1976). The clock may be monitored by successive determinations of critical flicker fusion (CFF) threshold or by a regularly recurring psychomotor task for its detection. These alternative monitors have been found to yield identical frequencies (Lovett Doust \& Podnieks, 1975). We have reported finding a spontaneous ultradian neural rhythm of $5.82 \mathrm{~min}, \mathrm{SD}=1.15$, in a group of 27 adult healthy subjects examined cross-sectionally (Lovett Doust \& Podnieks, 1976).

Pittendrigh (1960) has summarized 16 characteristics of circadian-length biological clocks, 8 of which would also relate to those with ultradian frequency. In confirmation of the CFF findings of our crosssectional group, a new series of subjects have now been studied longitudinally in an attempt to determine how closely our perceptual clock approximates the properties of the free-running rhythms investigated by Pittendrigh.

We gratefully acknowledge the Linc-8 computer assistance given by Mr. Ravi Sadana and Mr. David Payne, MSc, of the Clarke Institute Computer Service. We also thank the many physicians of the Hospital staff for referring patients to us.

\section{METHODS}

\section{Subjects}

Eight healthy subjects, four male and four female, aged 24 to 55 years (mean 35.7) were each tested on 20 occasions (total $N=160$ ). Each subject was tested daily at the same time and under similar conditions 5 days per week for a total of 20 days. The same room, low level of illumination, and air-conditioning controls were employed. Before the commencement and at the completion of each day's testing, oral temperature was measured in each subject by means of a clinical thermometer placed sublingually. Each subject was asked to keep a daily diary.

Three male and seven female neuropsychiatric patients aged 20 to 74 years (mean 40.1 ) were similarly tested on 292 occasions. Included in this group were five patients with structural disease of the CNS: two of these suffered from epilepsy, one from postencephalitic brain syndrome, one from cerebral atherosclerosis, and one patient who had been lobotomized for manic depressive psychosis. They were examined on a total of 276 occasions. In addition, there were four psychiatric patients who were physically well: two suffered from bi-polar manic depressive psychosis, one from chronic schizophrenia, and one from chronic anxiety state. They were examined on a total of 13 occasions. The remaining patient suffered from hypothyroidism and was examined on three occasions before and during treatment.

Daily observation was made of the patient's mental state and behavior by nurses and clinical staff. Each of these 10 patients was treated by appropriate drugs and the prescription and dosage of these drugs was altered by the attending psychiatrist to meet the patient's changing needs. Advantage was taken by us of the drug changes to retest the CFF and thus check the setting of the perceptual clock. In all, a total of 18 different drugs were employed in the treatment of these patients over a period of about 18 months; they ranged from anti-epileptics to hypnotics, from anti-depressants to hormones, and from tranquilizers to psycho-stimulants.

Each patient's drug regimen at the times of serial testing has been isolated for the purpose of computing our results and separated from his next medication change into a series of "drug phases." Thus, a patient might be examined over a period of days without medication in Phase I, and then be placed on a tranquilizing drug in drug Phase II, and the examinations continued. If now the treatment demands that the tranquilizing drug be replaced by an anti-depressant, the continued testing 
would now be deemed drug Phase III, etc. The no-drug phase and relevant drug phases CFF periodicities, are listed for each patient in Table 2.

\section{Critical Flicker Fusion (CFF) Threshold}

Testing was carried out without comment by the examiner employing a technique already described (Lovett Doust \& Coleman, 1955), using a semidarkened room, with the subject seated $1.5 \mathrm{~m}$ from a Stroboscope masked to provide a circular white-light patch $2.5 \mathrm{~cm}$ in diameter. Two runs, totaling $30 \mathrm{sec}$, were then made each minute, for a duration of $20 \mathrm{~min}$, and the flash rates at which the light was first perceived as intermittent and at which it was first perceived as constant were recorded. The "mean score CFF" for each minute was then separately plotted minute by minute against time on graph paper (Figure 1).

\section{Effects of Repetition of This Test}

When the individual mean scores of the CFF are plotted separately against time, spontaneous endogenous cycles of recurrence emerge in the individual time series. With a test repetition interval of $60 \mathrm{sec}$, the periodicity of these cycles is measurable in minutes. A second necessary component of any sine-wave cycle is amplitude, and this may be conveniently estimated in hertz units from the graphs. Cycle periodicity was determined statistically by an adaptation of autocorrelation analysis (Kendal, 1948) to the Linc-8 computer (Southworth, 1967) by a program designed by $D$. Payne of the Clarke Institute Computer Service. Lags 1-10 were used for each individual's results with the maximum lag of $10 \mathrm{~min}$.

Autocorrelation has been defined as the degree of interdependence between the scores obtained at different equally spaced points in a time series. In our case, scores separated by 1 min of time can yield an autocorrelation coefficient by computing the product moment correlation coefficient between pairs of lags. This autocorrelation coefficient at Lag 1 is thus an index of the extent of the correlation existing at the time of Lag 1 and that at Lag 2, $60 \mathrm{sec}$ later. In a regular time series, its autocorrelation is an index of the predictability of one set of scores from a preceding set. An x-y plot of the autocorrelation functions with their appropriate lags is known as a correlogram, and this may be used as a means of identifying the maxima and the minima of a recurrent cycle. The statistical significance of a cycle, the existence of which has been predicted by a series of autocorrelations, may be assessed from the correlation coefficient probability tables where $r$ for each lag is determined by the degree of freedom minus the lag being examined [(N - 2) - lag $\mathrm{x}]$. Huba, Lawlor, Stallone, and Fieve (1976) have recently published an account of the application of autocorrelation analysis to the recurrent mood swings of 10 hospitalized psychiatric patients. Their study revealed the existence of "mini cycles" of cyclothymic changes, the regularity of which was not clinically obvious before this technique was used.

Autocorrelation procedures, when applied to the 20-day observations on the eight healthy subjects, yielded statistically significant spontaneous endogenous cycles in 19 of the 20 daily tests in one subject, in another in 18,16 in five subjects, and 15 in the remaining one. Thus the total of 160 timed tests yielded 132 perceptual cycles, an incidence of $82.5 \%$. In the case of the 10 patients and their 292 timed tests, the number of significant cycles was 174, a somewhat lesser incidence of $59.59 \%$. If the observations had been continued beyond the 20 min allowed, it was felt that significant cycles might have appeared in an even higher proportion of cases. While it is acknowledged that the description of an ultradian periodicity of ca 5 to $10 \mathrm{~min}$ by autocorrelation analysis might require a testing time of upwards of $1 \mathrm{~h}$ or more, the 20-min timed test used in this study was a compromise between that ideal and the practical cooperation to be expected from a group of psychiatric patients. No reward or additional motivation was offered to any subject or patient to insure his cooperation with the procedure, and the cycles, where present, appeared spontaneously and often revealed themselves when plotted on graph paper (Figure 1).

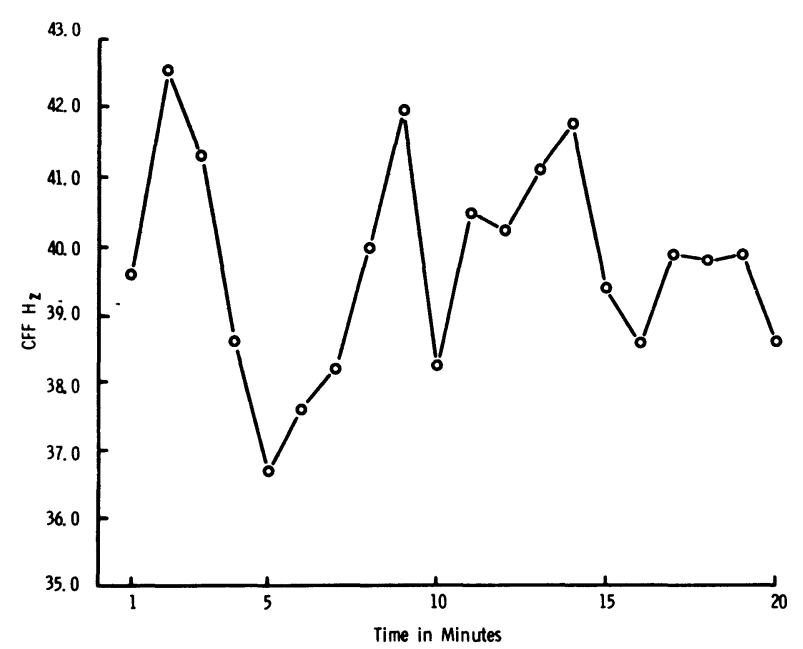

Figure 1. Miss L.M.D., aged 27, control subject. Minute-byminute CFF mean scores during a typical 20-min test. Two spontaneous sine-wave cycles are evident, the first with a period of $8 \mathrm{~min}$ and the second with a period of $7 \mathrm{~min}$. During her 20-day series of timed tests, Miss D's cyclic periodicity ranged between 4 and $10 \mathrm{~min}$, with a mean of $7.35 \mathrm{~min}$.

\section{RESULTS}

Our results cover the 306 statistically significant cycles revealed in the present study and refer principally to the periodicity of these cycles. It is, however, relevant that their amplitude bears a significant relationship to this periodicity $(\mathrm{r}=.164, \mathrm{df}=304$, $\mathrm{p}=.05$ ). Evidence in support of Pittendrigh's eight properties is given below. Tables 1 and 2 summarize the results.

Plotting the successive minute-by-minute CFF mean scores against time for the total of $20 \mathrm{~min}$ and subjecting these to autocorrelation analysis for each of the individual healthy subjects yielded statistically significant $(\mathrm{p}=.05$ or better) cycles of recurrence for each subject. Mean cycle length for the 132 serial timed tests which contained significant periodicities in the healthy subjects was $6.49 \mathrm{~min}, \mathrm{SD}=.52$.

Despite their varying occupations, marital relationships, interactions with diverse environments, and

Table 1

Analysis of CFF Cycle Frequencies Taken from Eight Healthy

Subjects Each Tested for 20 Days During a 4-Week Period

\begin{tabular}{lccr}
\hline & $\begin{array}{c}\text { Number of } \\
\text { Serial Tests }\end{array}$ & $\begin{array}{c}\text { Mean } \\
\text { Cycle } \\
\text { Frequency } \\
\text { Healthy Subjects }\end{array}$ & \\
\hline Yielding Cycles & (Minutes) & SD \\
Mr. G.C. & 18 & 6.17 & 1.15 \\
Mr. J.L.D. & 16 & 6.18 & .98 \\
Mr. J.M. & 16 & 6.68 & .70 \\
Mr. I.P. & 16 & 7.00 & .82 \\
Miss L.D. & 16 & 6.94 & 1.24 \\
Mrs. S.H. & 16 & 5.87 & 1.78 \\
Mrs. L.J. & 19 & 7.16 & 1.21 \\
Mrs. S.L. & 15 & 5.93 & .79 \\
Totals & 132 & 6.49 & .52 \\
\hline
\end{tabular}

Note-Of 160 CFF tests performed, 132 contained cycles. 
Table 2

Analysis of CFF Cycle Frequencies Taken from 10 Psychiatric Patients Each Tested Under Varying Treatment Conditions and for Various Numbers of Observations

\begin{tabular}{|c|c|c|c|c|c|c|c|c|c|c|c|c|c|c|c|c|c|c|}
\hline \multirow{3}{*}{$\begin{array}{l}\text { Psychiatric } \\
\text { Patients }\end{array}$} & \multirow{2}{*}{\multicolumn{3}{|c|}{$\begin{array}{c}\text { Drug Free } \\
\text { Phase I }\end{array}$}} & \multicolumn{15}{|c|}{ Drug Phases Representing Serial Prescriptions of Changing Drug Regimens } \\
\hline & & & & \multicolumn{3}{|c|}{ Phase II } & \multicolumn{3}{|c|}{ Phase III } & \multicolumn{3}{|c|}{ Phase IV } & \multicolumn{3}{|c|}{ Phase V } & \multicolumn{3}{|c|}{ Phase VI } \\
\hline & $\mathrm{C}^{*}$ & MCL* & * SD & $\mathrm{C}$ & MCL & SD & $\mathrm{C}$ & MCL & SD & $\mathrm{C}$ & MCL & SD & $\mathrm{C}$ & MCL & SD & $\mathrm{C}$ & MCL & SD \\
\hline & \multicolumn{18}{|c|}{ Nonorganic Patients } \\
\hline $\begin{array}{l}\text { Mrs. F.B. } \\
\text { Miss K.C. } \\
\text { Miss J.S. } \\
\text { Miss J.H. }\end{array}$ & $\begin{array}{l}2 \\
4 \\
2 \\
2\end{array}$ & $\begin{array}{l}6.00 \\
6.25 \\
6.50 \\
6.00\end{array}$ & $\begin{array}{r}1.15 \\
.96 \\
1.30 \\
1.20\end{array}$ & 2 & 5.50 & .53 & & & & & & & & & & & & \\
\hline & \multicolumn{18}{|c|}{ Organic Patients } \\
\hline $\begin{array}{l}\text { Mr. G.Z. } \\
\text { Mr. R.M. } \\
\text { Mrs. B.M. } \\
\text { Mr. M.R. } \\
\text { Mrs. E.M. } \\
\text { Mrs. B.B. }\end{array}$ & $\begin{array}{r}15 \\
4 \\
7 \\
9 \\
1 \\
12\end{array}$ & $\begin{array}{l}6.50 \\
4.75 \\
4.57 \\
5.40 \\
5.00 \\
5.2\end{array}$ & $\begin{array}{r}.58 \\
1.26 \\
1.13 \\
.88\end{array}$ & $\begin{array}{r}10 \\
4 \\
11 \\
7 \\
2\end{array}$ & $\begin{array}{l}6.90 \\
5.50 \\
4.64 \\
5.57 \\
4.5\end{array}$ & $\begin{array}{l}1.28 \\
1.29 \\
1.75 \\
1.72 \\
1.00\end{array}$ & $\begin{array}{r}12 \\
5 \\
5 \\
6\end{array}$ & $\begin{array}{l}5.50 \\
5.40 \\
4.40 \\
5.60\end{array}$ & $\begin{array}{l}1.56 \\
1.52 \\
1.34 \\
1.63\end{array}$ & $\begin{array}{l}9 \\
4 \\
8 \\
4\end{array}$ & $\begin{array}{l}5.60 \\
6.00 \\
4.62 \\
5.50\end{array}$ & $\begin{array}{r}1.27 \\
.82 \\
1.42 \\
1.29\end{array}$ & $\begin{array}{r}12 \\
4\end{array}$ & $\begin{array}{l}6.10 \\
4.50\end{array}$ & $\begin{array}{l}1.17 \\
1.29\end{array}$ & 11 & 5.10 & 1.04 \\
\hline
\end{tabular}

Note-Of 292 serial CFF tests performed, 174 contained cycles. Total CFF cycles nonorganic patients $N=12, M e a n=6.1, S D=.29$; organic patients $N=164$, Mean $=5.35, S D=.42$.

*Number of cycles

**Mean cycle length (in minutes)

changing emotional states, the perceptual cycles of members of this normal group were unchanged. Endogenous oscillations regulating sensory perception appear remarkably constant. No tuning by a possible Zeitgeber has disturbed their perceptual clock. A further proof of this is reflected in a splithalf reliability test of the series of 20 days of observation undertaken on each control subject. No significant differences between the first 10 days and the last 10 days were found for any subject.

Three of the eight subjects were females who menstruated regularly. The time of their menstrual periods could be identified with the variance of their actual CFF thresholds but not in any variation of the periodicities of this measure. Neither the feeling states nor the intercurrent events reflected in the subject's diaries bore any correlatable relation with the CFF cycles found. In similar fashion, no relationship existed between external events and behavioral reactions of the psychiatric patients, observed by nursing staff and others, and the periodicities. No cognitive or affective Zeitgebers appear relevant.

The patients provided many examples in which the actual CFF mean scores varied greatly with their feeling states, e.g., G. Z. swung a low of $48.8 \mathrm{~Hz}$ in drug Phase IV to a high of $52.9 \mathrm{~Hz}$ in drug Phase V, while, as Table 2 suggests, there was no significant difference $(t=1.12, p=n . s$.$) between$ the cycle lengths of these two drug phases. Another example was provided by F. B., who was examined on two occasions while depressed and on another two occasions while hypomanic. At the time of her endogenous depression (no-drug Phase I), her perceptual mean cycle length was $6.0 \mathrm{~min}, \mathrm{SD}=1.15$. During the time of hypomania (drug Phase II), the mean $\mathrm{CFF}$ cycle length was $5.5 \mathrm{~min}, \mathrm{SD}=.53$.
There was no statistically significant difference between the cycle frequencies of these polar moods. R.M. was examined while depressed (no-drug Phase I and drug Phase III) and while in remission (drug Phase II and IV). Analysis of variance indicated that no differences existed between the cycle frequencies $[F(4,16)=.90, p=n . s$.$] . Another$ example is that of patient B.M., whose mood underwent recurrent changes. Her hypomanic mood (drug Phases II and IV) would alternate with one of depression (no-drug Phase I and drug Phase III). Despite her often violent responses, an analysis of variance indicated no statistically significant differences between them for CFF mean cycle length $[\mathrm{F}(3,27)=.03, \mathrm{p}=\mathrm{n} . \mathrm{s}$. $]$. However, the noncyclic actual CFF mean score measurements in this patient did distinguish $(p<.01)$ between the hypomanic (mean CFF, 31.26 Hz; SD = 3.22) and depressed (mean CFF, 35.0 Hz; SD = 2.39).

The autocorrelation analyses of 132 of the 160 timed observations during the months of testing of the eight healthy subjects contained statistically significant spontaneous oscillations of the CFF scores. Similarly, 174 statistically significant self-sustaining cycles were found among the 292 serial timed observations on the 10 patients.

Pittendrigh's assumption that biological clocks occur at levels of complex organization as well as at cellular level is exemplified by our demonstration that the sensory perceptual clock we have described has its setting changed if neurological disorder has occurred in the CNS. Comparing the CFF mean cycle length of the eight healthy controls ( $\mathrm{N}$ of significant cycles $=132: 6.49 \mathrm{~min}, \mathrm{SD}=.52$ ) with those of the four neurologically intact and only emotionally sick patients $(\mathrm{N}$ of significant cycles $=12$ : mean CFF 
periodicity of $6.1 \mathrm{~min}, \mathrm{SD}=.29$ ), no significant difference existed. Comparison, however, between these healthy controls and the neurologically damaged patients $(\mathrm{N}$ of significant cycles $=164$ : mean CFF periodicity, $5.35 \mathrm{~min}$, SD .42) yielded a significant difference $(p<.02)$, as did the comparison between the neurologically intact and organically damaged groups $(\mathrm{p}<.02)$.

Another aspect of entrainment is that of the Zeitgeber. In our experience of the ultradian periodicities of our sensory perceptual clock, the phase of the clock responds predictably to changes in the "set" of the subject and to the focusing of his state of awareness at any given time. In numerous instances, we have deliberately intervened during the minute-by-minute CFF testing and engaged the subject in conversation for varying lengths of time. On resuming the minute-by-minute silent testing for the balance of the $20 \mathrm{~min}$, it has been found that the subject has reverted to his original starting point, thus interrupting the otherwise continuous repetition of his sine-wave perceptual clock. Figure 2 illustrates this phenomenon in a control subject.

The daily 20-min tests of each of our eight healthy subjects showed remarkably little variation both among the series for each subject and between all the members of this group. The group range of the CFF cycles for all of the eight subjects lay between 7.17 and $5.88 \mathrm{~min}$, and the SD between 1.78 and .70 , respectively. A split-half reliability test provided a check of this variance. There was no statistically significant difference between the mean CFF cycles of the first 10 days of testing and that of the second 10 days for any subject $(p=n . s$.).

This property applies equally to the patients. Taking those four patients (G.Z., R.M., B.M., and M.R.) who were investigated most intensively, the following means and standard deviations apply. The range of the mean CFF cycles for all four patients lay between 5.77 and $4.58 \mathrm{~min}$ and the SD between 1.41 and 1.26, respectively. A split-half reliability test provided a check of this variance.

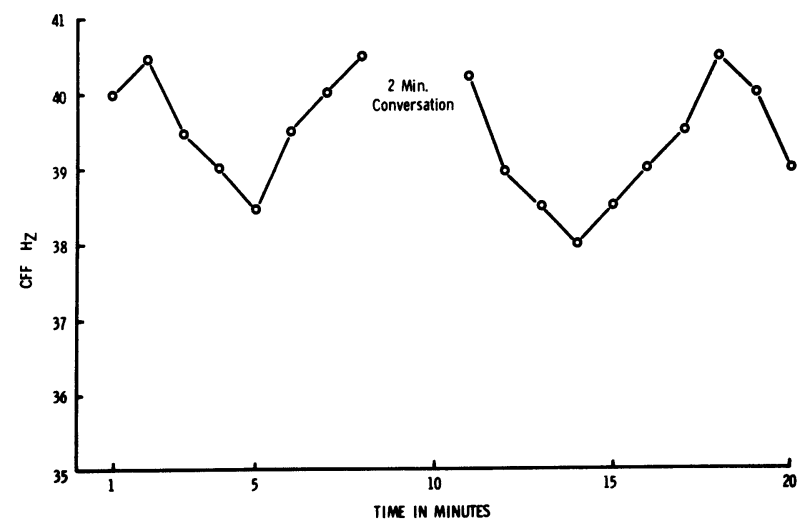

Figure 2. Effect of the phase of the ultradian rhythm of challenging a healthy subject's attention during the course of testing. Subject Miss L.M.D., aged 27.
There was no statistically significant difference between the mean CFF cycles of the first half of each patient's testing occasion and that of the second half $(\mathrm{p}=$ n.s.).

All investigations were conducted in an airconditioned room at an ambient temperature held constant at $21^{\circ} \mathrm{C}$. Body temperature was recorded before each 20 min testing for all 20 days of testing in all eight healthy subjects. Correlation coefficients between body temperature and mean score CFF among the eight healthy subjects ranged between .09 and $.41(\mathrm{p}=$ n.s.), and between body temperature and mean score CFF cycles from .04 and .33 $(\mathrm{p}=$ n.s. $)$.

Of the wide variety of chemicals employed on patients monitored in this investigation (18 drugs in all), only the anticonvulsants sometimes appeared to be capable of influencing the perceptual clock. As Table 2 indicates, in all other instances where one patient's phase could be compared with another in which a different drug regimen had been given, this change in drugs did not alter the perceptual periodicity. One example is F.B., whose perceptual cycle length was not changed when methylphenidate $\mathrm{HCl}$ and amobarbital sodium were administered in drug Phase II. Another example is that of R.M., where the addition of drugs in drug Phase II did not disturb the CFF periodicity of the no-drug condition of no-drug Phase I. One further example is that of the post-leucotomy patient, B.M. Despite the four different chemical substances used in the various phases of her treatment, no significant differences in the periodicity of her sensory perceptual cycle was found to exist $[F(3,27)=.03, p=n . s$.$] .$

Our results include one patient, M.R., who was given anticonvulsants in all phases of his treatment. In this patient, analysis of variance showed no difference between the CFF mean periodicities $[F(3,22)=$ $.03, p=n . s$.$] . Where the anticonvulsant appears in$ four phases but not in the remaining two (G.Z.), the difference between the means is statistically significant $[F(5,63)=3.76, p<.01]$. On the other hand, as in the case of R.M., diphenylhydantoin was exhibited only during one of his drug phases, resulting in no significant difference between the CFF mean cycles $[F(4,16)=.90, p=n . s$.$] .$

Aside from the aberrant results of anticonvulsant drugs, therefore, it would appear that none of the wide range of chemicals used here were able to influence the timing of the patients' perceptual clocks.

\section{DISCUSSION}

William Goody (1956) stated that the passage of time is essential for perception. He claimed (1956, 1958) that man must be equipped with internal chronometric apparatus amenable to physiological analysis. Evidence for the existence of a perceptual clock, its frequency in healthy man and the periodi- 
cities showing acceleration in one form of neurological deficit (epilepsy) over those of neural integrity have been provided in terms of microcirculatory dynamics and autonomic effectors (Lovett Doust, $1960,1964)$. More recently, we have adduced the existence of the perceptual clock from cross-sectional studies of neurologically healthy and brain-damaged adult patients (Lovett Doust \& Podnieks, 1976) and from healthy and mentally retarded children (Podnieks \& Lovett Doust, 1975).

The present investigation is a longitudinal one [in accordance with Chassan's (1967) "intensive" technique] on a further series of subjects permitting a more detailed study of the properties of this perceptual clock.

We have investigated a neural clock specific for sensory perception. We have indicated in our examination of the properties of this clock in healthy subjects that it possesses every relevant characteristic of the biological clock studied by Pittendrigh (1960) in his consideration of circadian rhythms. Those relevant properties include: such a clock is endogenous and innate; perceptual rhythms are self-sustaining oscillations; these rhythms are dependent upon neural integrity and are entrainable by damage to the neural clock; the phase of a free-running rhythm can be shifted by a single perturbation: the perceptual clock has a very small variance and exhibits precision; these periodicities are independent of temperature; and the perceptual rhythms are relatively intractable to chemical perturbations. This perceptual clock maintains these properties despite intercurrent events and the reactive feeling states on the part of eight healthy subjects during the 160 daily occasions when they were examined. We have further shown that these properties are equally seen in the case of four physically healthy psychiatric patients during their 13 repetitions of these tests. These "functional" psychiatric disturbances in no way altered the periodicity of the clock characterizing the sensory perception of the healthy subjects. The cyclic characteristics of the clocks of the functionally sick patients were indistinguishable from those of the controls despite their different emotional status, their diagnosis, and the many chemical substances prescribed for their treatment. Qualitatively different from these findings, however, were those of the six remaining patients. Five of these had suffered organic lesion to the brain of one sort or another. The sixth was a patient whose hospitalization was necessitated by hypothyroidism. All these patients had suffered injury to the integrity of their CNS. The pathology may have been structural and permanent or biochemical and transitory. Impaired brain function showed itself in the new setting of the neurological patient's perceptual clock. CFF mean periodicity was shorter at a frequency of $5.35 \mathrm{~min}, \mathrm{SD}=.42$, than for the controls $(6.49 \mathrm{~min}$, $\mathrm{SD}=.52$ ) and neurologically normal patients
(6.1 min, $\mathrm{SD}=.29)$. Amplitude was also reduced in the brain-damaged patients. These observations relating to damaged brain function were as stable as those of the healthy CNS and were not influenced to any significant extent by many variables, including drugs employed for treatment.

Goody (1958) stated that those processes loosely referred to as memory, foresight, judgment, intelligence, behavior, and concentration are disordered in cases where damage to parts of the cerebral cortex exist. All these processes involve timing. Perception depends on the spatiotemporal arrangement of nervous activity. Kleitman (1963) has postulated a rest-activity cycle revealed by the repetitive REM periods of sleep. This cycle, with a periodicity of 90-100 min, has been demonstrated by Kripke (1972) and its existence confirmed by Lavie, Levy, and Coolidge (1975).

The perceptual clock appears to us to be not primarily cortical, however, but to lie deep within the neurons of the midbrain, since it regulates the operation of the ascending reticular activating system and may be subject to the rhythmicity of the lateral hypothalamus (Lovett Doust, 1960) or the nucleus suprachiasmaticus (Stetson \& Watson-Whitmyre, 1976). It has been claimed (Bowen \& Skopik, 1976) that feedback mechanisms do not explain the time periods of all biological cycles. Currently, the most attractive hypothesis underlying many basic clock mechanisms seems to be that of the changing permeability of cell membranes to ionic fluxes (Jones \& Mansfield, 1975; Njus, Sulzman, \& Hastings, 1974).

\section{REFERENCE NOTE}

1. Luce, G. G. Biological rhythms in psychiatry and medicine. U.S. Public Health Service Publication No. 2088, Washington, D.C., 1970.

\section{REFERENCES}

Aschoff, J. Human circadian rhythms in activity, body temperature and other functions (Symposium). In A. H. Brown \& F. G. Favorite (Eds.), Life science symposium, 7th, Vienna, Austria, May 10-18, 1966. Amsterdam: North Holland, 1967. Pp. 159-173.

Bowen, M. F., \& Skopik, S. D. Insect Photoperiodism: The "T experiment" as evidence for an hourglass mechanism. Science, 1976, 192, 59-60.

Chassan, J. B. Research design in clinical psychology and psychiatry. New York: Appleton-Century-Crofts, 1967.

Goody, W. Cerebral representation. Brain, 1956, 79, 167-187.

Goody, W. Time and the nervous system: The brain as a clock. Lancet, 1958, 1, 1139-1144.

Huba, G. J., Lawlor, W. G., Stallone, F., \& Fieve, R. R. The use of autocorrelation analysis in the longitudinal study of mood patterns in depressed patients. British Journal of Psychiatry, 1976, 128, 146-155.

Jones, M. B., \& Mansfield, T. A. Circadian rhythms in plants. Science Progress, Oxford, 1975, 62, 103-125.

KENDALL, M. G. The advanced theory of statistics. London: Griffin, 1948.

Kleitman, N. Sleep and wakefulness. Chicago: University of Chicago Press, 1963. 
KRIPKE, D. F. An ultradian biological rhythm associated with perceptual deprivation and REM sleep. Psychosomatic Medicine, 1972, 3, 221-234.

Lavie, P., Levy, C. M., \& Coolidge, F. L. Ultradian rhythms in the perception of the spiral aftereffect. Physiological Psychology, 1975, 3, 144-146.

Lovetr Doust, J. W. Spontaneous endogenous oscillating systems in autonomic and metabolic effectors: Their relation to mental illness. Journal of Nervous and Mental Disease, 1960, 131, 335-347.

Lovetr Doust, J. W. Biological clocks: Psychophysiologic evidence for the existence of one such clock specific to certain psychiatric disorders. Canadian Psychiatric Association Journal, 1964, 9, 210-220.

Lovett Doust, J. W., \& Coleman, L. I. M. The psychophysics of communication. American Medical Association Archives of Neurology and Psychiatry, 1955, 74, 650-652.

Lovett Doust, J. W., \& Podnieks, I. Comparison between some biological clocks regulating sensory and psychomotor aspects of perception in man. Neuropsychobiology, 1975, 1, 261-266.

Lovett Doust, J. W., \& Podnieks, I. Two biological rhythms of perception distinguishing between intact and related damaged brain function in man. International Journal of Chronobiology, 1976, in press.
Nuus, D., Sulzman, F. M., \& Hastings, J. W. Membrane model for the circadian clock. Nature, 1974, 248, 116-120.

Pittendrigh, C. S. Circadian rhythms and the circadian organization of living systems. In A. Chovnick (Ed.), Cold Spring Harbor symposia on quantitative biology (Vol. 25) Biological clocks. Cold Spring Harbor, L. I., New York: Long Island Biological Association, 1960. Pp. 159-184.

Podnieks, I., \& Lovetr Doust, J. W. Emergence of a biological rhythm when certain psychological tests are repeated at regular intervals in mentally retarded children. Journal of Interdisciplinary Cycle Research, 1975, 6, 213-222.

Southworth, R. W. Autocorrelation and spectral analysis. In A. Ralston \& H. S. Wilf (Eds.), Mathematical methods for digital computers (Vol. I). New York: Wiley, 1967. Pp. 213-220.

Stetson, M. H., \& Watson-Whitmyre, M. Nucleus suprachiasmaticus: The biological clock in the hamster? Science, 1976, 191, 197-199.

(Received for publication May 28, 1976; revision accepted October 7, 1976.) 